Klinichna khirurhiia. 2018 March;85(3):39-42.

DOI: $10.26779 / 25221396.2018 .03 .39$

УДК 616-056.52-089.168

\title{
Сравнительная оценка результатов различных хирургических бариатрических операций
}

\section{Т. Омаров, С. Салахова, А. Ибрагимова, Н. Байрамов \\ Азербайджанский медицинский университет, г. Баку, \\ Модерн клиника, г. Баку, Азербайджан \\ Comparative estimation of results of various surgical bariatric operations}

\author{
T. Omarov, S. Salakhova, A. Ibrahimova, N. Bayramov \\ Azerbaijani Medical University, Baku, \\ Modern Clinic, Baku, Azerbaijan
}

\section{Реферат}

Цель. Сравнить результаты выполнения за 5 лет бариатрических хирургических операций у пациентов с ожирением среди населения Азербайджана.

Материалы и методы. Бариатрические хирургические операции выполнены у 104 пациентов с ожирением среди населения Азербайджана. Средний возраст пациентов - 33,1 года, средний индекс массы тела (ИМТ) - 57,5 кг/м². Проведен сравнительный анализ различных технических модификаций, послеоперационных осложнений. Больных обследовали до и через 1, 3, 6 и 12 мес после операции. В динамике исследовали ИМТ, проявления диабета, гипертонии, синдрома апноэ во сне и жировой дистрофии печени.

Результаты. У 88 (84,6\%) пациентов, которым выполнена стандартная лапароскопическая СлИВ гастрэктомия (ЛСГ) в течение первых 6 мес масса тела уменышилась в среднем на $(39,5 \pm 11,5)$ кг. У $12(11,5 \%)$ больных после желудочного шунтирования (2 (1,9\%) выполнено желудочное шунтирование по Ру, 8 (7,6\%) - желудочное минишунтирование, 2 (1,9\%) - СЛИВ и шунтирование по Ру) масса тела уменьшилась на (46 \pm 14$)$ кг, а в течение 6-12 мес наблюдали эффективное ее увеличение, в среднем на $(33,5 \pm 8,5)$ кг. У 1 (1\%) больного была выполнена в другой клинике дупликация желудка. Масса тела за год уменьшилась на 20 кг и остановилась. В связи с этим больной обратился в нашу клинику. Ему выполнена операция уменышения желудка. Масса тела уменышилась на 36 кг. У 3 (2,9\%) больных, которые по истечении 2 лет после операции набрали массу тела, повторно выполнили СлИВ гастрэктомию. В последующем у этих больных отмечено удовлетворительное уменышение массы тела.

Выводы. После ЛСГ уменьшение массы тела у больных с тяжелым ожирением наиболее удовлетворительное, также отсутствуют нарушения витаминного баланса, динамика сопутствующей патологии положительная, что дает основание считать этот способ наиболее надежным. Однако после операции шунтирования упомянутые показатели выше и при диабете II типа эта методика наиболее эффективна. Но с учетом анатомии и физиологии СЛИВ гастрэктомия является идеальной операцией, поскольку при неэффективном снижении массы тела в будущем есть возможность применить другую методику, кроме того, эта операция также приемлема для больных, у которых нет диабета II типа.

Ключевые слова: тяжелая форма ожирения; СлИВ гастрэктомия; желудочное шунтирование; антрумэктомия; пилорический сфинктер.

Abstract

Objective. To compare the results of the bariatric surgical operations for the 5 year period in patients, suffering obesity among population of Azerbaijan.

Materials and methods. Bariatric surgical operations were performed in 104 patients, suffering obesity among population of Azerbaijan. Average age of the patients was 33.1 years old, and average body mass index (BMI) $-57.5 \mathrm{~kg} / \mathrm{m}^{2}$. Comparative analysis of various technical modifications and postoperative complications was conducted. The patients were examined preoperatively and in 1, 3, 6 and 12 mo after the operation. BMI, the signs of diabetes, hypertension, the apnoe syndrome while sleeping and hepatic adipose dystrophy were investigated in dynamics.

Results. In 88 (84,6\%) patients, in whom a standard laparoscopic sleeve gastrectomy (LSG) was performed, in 6 mo afterwards the body mass have reduced by $(39.5 \pm 11.5) \mathrm{kg}$ at average. Of $12(11.5 \%)$ patients after gastric shunting in $2(1.9 \%)$ patients a gastric shunting in accordance to Roux method was performed, in 8 (7.6\%) - gastric minishunting, 2 (1.9\%) - LSG and Roux shunting. As a result, the body mass have reduced by $(46 \pm 14) \mathrm{kg}$, and during $6-12$ mo observation its effective increase was observed by $(33.5$ $\pm 8.5) \mathrm{kg}$ at average. In 1 (1\%) patient in other clinic the gastric duplication operation was performed. The body mass during the following year have reduced by $20 \mathrm{~kg}$ and stopped, so the patient have been applied into our clinic and to him gastrorestrictive operation was performed. His body mass have reduced by $36 \mathrm{~kg}$. In $3(2.9 \%)$ patients, who have gained the body mass in 2 years after the operation, reoperation procedure was LSG. Subsequently in these patients a satisfactory reduction of the body mass was noted. Conclusion. After LSG performance the body mass reduction in patients with severe obesity is mostly satisfactory, the vitamin balance disorders are absent, and dynamics of concurrent pathology is positive, making this procedure mostly secure. Meanwhile after shunting bariatric surgery the abovementioned indices are higher and in presence of diabetes mellitus this procedure is mostly effective. LSG is anatomically and physiologically ideal operation, because in case of ineffective body mass lowering the reoperation is possible, and this operation is feasible in patients without diabetes Type II.

Keywords: severe form of obesity; sleeve gastrectomy; gastric shunting; antrumectomy; pyloric sphincter. 
Согласно данным ВОЗ за 2011 год в мире избыточной массой тела и метаболическим синдромом страдали более 2,1 миллиарда человек, к 2025 году этот показатель удвоится. Данные статистики свидетельствуют, что в Азербайджане число пациентов этой категории довольно велико.

Ожирение является хроническим, многосистемным заболеванием, которое приводит к возникновению нарушений венозного кровообращения, диабета II типа, гипертонии, гиперкоагулопатии, неалкогольной жировой болезни печени и дефектов репродуктивной системы, а также ортопедических осложнений, артрита, рака толстой кишки, молочной железы и печени. Согласно данным Национального центра здравоохранения США у 60\% больных с ожирением снижение массы тела после диет и физической нагрузки держится всего год. Через 5 лет почти у всех пациентов масса тела возвращается к начальным показателям, а в некоторых даже превышает их. В то же время при консервативном лечении ожирения многие сопутствующие заболевания не проходят, например, диабет II типа. Поэтому крайне необходимо применять хирургическую коррекцию. Выбор типа операции зависит от состояния больного и сопутствующей патологии. После хирургической коррекции ожирения резистентность к инсулину снижается, а проявления жировой болезни печени, гипертонии и патологии дыхательной системы уменьшаются примерно на 90\%.

Первые данные об операциях при ожирении восходят к 1950-м гг, сообщения о более эффективных хирургических процедурах начали появляться после 1979 года $[1-3]$.

Однако прорыв в бариатрико-метаболической хирургии произошел после принятия в 1991 г. ВОЗ концепции о только хирургической коррекции этой патологии, после чего ожирение во всем мире было включено в перечень хирургических заболеваний. Было рекомендовано использовать для оценки состояния этих пациентов ИМТ. Пациентов с ИМТ более 35 кг/м² следует лечить хирургически, менее - консервативно [4].

Основная концепция бариатрической хирургии заключается в том, что для наиболее эффективного лечения ожирения необходимо снижение аппетита и всасываемости из желудочно-кишечного тракта, что достигается выполнением или операции для снижения мальабсорбции, или операции уменьшения желудка (рестриктивные операции) либо эти операции выполняют одновременно [5, 6].

Целью бариатрической хирургии, наряду с уменышением массы тела, является также устранение сопутствующей патологии. Мы наиболее часто используем СлиВ гастрэктомию, ЛСГ или операцию уменьшения желудка, которая является относительно новым хирургическим методом. В среднем объем желудка уменьшают до 100-120 мл. Хотя основным механизмом похудения является уменьшение объема желудка, важную роль в снижении массы тела и нормализации метаболических расстройств играет нарушение синтеза гормона грелина. Во время операции желудок резецируют по большой кривизне до гастроэзофагеального соединения, после чего секреция этого гормона невозможна, аппетит уменьшается, что приводит к эффективному здоро- вому снижению массы тела. В настоящее время ЛСГ считают наиболее распространенным типом операции при ожирении $[7,8]$.

При лапароскопической операции желудочного шунтирования по Ру желудок резецируют проксимально с помощью степлера, образуется желудочный резервуар объемом около 20 мл. Тонкую кишку на 50 - 70 см проксимальнее от Трейтцевой связки разрезают и создают анастомоз с желудочным резервуаром в 1 см. Проксимальную часть тонкой кишки анастомозируют в дистальной части 75 - 150 см в зависимости от степени ожирения пациента.

При операции желудочного минишунтирования, или наложения одного анастомоза, желудок зашивают проксимально с помощью степлера параллельно малой кривизне, образуя желудочный резервуар объемом приблизительно 60 - 80 мл. Дистальнее на 200 - 250 см от Трейтцевой связки петлю тонкой кишки поднимают и создают анастомоз с желудочным резервуаром в 1 см [9].

Цель исследования: сравнить результаты бариатрическо-метаболических хирургических операций различной модификации, выполненных нами у пациентов с избыточным ожирением среди населения Азербайджана.

\section{Материалы и методы исследования}

За период с 2012 по 2017 г. в Модерн клинике и Учебно-хирургической больнице Азербайджанского медицинского университета оперированы с применением различных лапароскопических методов 104 больных с ожирением. Средний возраст больных - 33,1 года, средний ИМТ - 57,5 кг/м². Женщин было 86 (82,7\%), мужчин - 18 (17,3\%). У 43 (41,3\%) больных выявлен диабет II типа, у 33 (31,7\%) - гипертензия, у 19 (18,2\%) - апноэ сна, у 17 (16,3\%) - гормональная дисфункция, связанная с поликистозом яичников, у 5 (4,8\%) - нарушение половой активности, у 14 (13,4\%) - дегенеративный остеоартрит, у 1 (1\%) - хроническая обструктивная болезнь легких. У 2 (1,9\%) больных ранее выполнена операция установки коронарного стента по поводу ишемической болезни сердца. У 98\% пациентов була жировая дистрофия печени IV степени.

Хирургические показания разработаны на основе рекомендаций IFSO (International Federation for the Surgery of Obesity and Metabolic Disorders) 2006 года. Перед операцией учитывали массу тела, ИМТ и сопутствующие заболевания. До операции всем пациентам для оценки состояния желудочно-кишечного тракта проводили гастроскопию, для оценки состояния печени, желчных путей - ультразвуковое исследование. Всех пациентов консультировали пульмонолог, кардиолог, диетолог, психолог и эндокринолог, а также анестезиолог для оценки анестезиологического риска. До и после операции применяли фракционный гепарин, на ноги больных надевали эластичные носки, дополнительно использовали динамические массажеры для ног. С целью профилактики назначали антибиотики цефалоспориного ряда: до операции - одну дозу, после - две.

Проведенные операции в зависимости от технических модификаций разделены на три группы. После стандартных дооперационных мероприятий 88 (84,6\%) 
больным выполнена СЛИВ гастрэктомия, 2 (1,9\%) - желудочное шунтирование по Ру, 8 (7,6\%) - желудочное минишунтирование, 1 (1\%) СлИВ и желудочное шунтирование по Ру, 1 (1\%) - рукавная гастрэктомия и желудочное минишунтирование, 1 (1\%) - операция уменьшения желудка после его дупликации (складывания) в другой клинике (за год масса тела снизилась на 20 кг и остановилась). У 3 (2,9\%) больных, которые по истечении 2 лет набрали массу тела, выполнили повторную СлиВ гастрэктомию, при динамическом наблюдении в течение года результаты удовлетворительные. Технически операции выполнены в соответствии с международными стандартами. Желудочное шунтирование по Ру и желудочное минишунтирование проводили по стандартной методике. СЛИВ гастрэктомию технически выполняли иначе. Изучение сообщений в мировой литературе показало, что использование калибровочного зонда диаметром 42 Fr не влияет на снижение массы тела в течение 6 мес. Исходя из удовлетворительных отдаленных результатов, целесообразно использовать калибровочный зонд диаметром 32 Fr. В то же время исследователи рекомендуют выполнять резекцию на расстоянии 4 - 6 см от пилорического сфинктера. Мы во время операции использовали калибровочный зонд диаметром 32 Fr и, отступив 2 см от пилорического сфинктера, по телу желудка к его дну параллельно малой кривизне завершали резекцию с помощью 60-миллиметрового линейного степлера. Применяя эти два метода, формировали уменьшенный желудок, что приводило к более серьезному и долгосрочному снижению массы тела. На следующем этапе в желудок вводили метиленовый синий для контроля линии степлера. Интраоперационный контроль выполняли с целью профилактики несостоятельности швов анастомоза: дополнительно накладывали швы по линии степлера, нередко вместе с оментопексией, чтобы препятствовать скручиванию желудка диаметром приблизительно в 1 см. Всем пациентам с целью контроля возможной несостоятельности линии степлера проводили дренирование брюшной полости. Резецированный желудок удаляли через 15-миллимитровый троакар.

Операции у 2 (1,9\%) больных проведены открытым, у 102 (98\%) - лапароскопическим способом. Средняя продолжительность операции составила $(2,5 \pm 0,5)$ ч, средние сроки лечения в стационаре - $(2,5 \pm 0,5)$ дня. Все больные живы.

\section{Результаты}

В послеоперационном периоде изучены трудности различных модификаций операций, дана сравнительная оценка, также в динамике в 1, 3, 6 и 12 мес контролировали ИМТ, показатели артериального давления и проводили специальные методы исследования у больных с жировой дистрофией печени.

У 1 (1\%) больного через 4 дня из-за несостоятельности швов анастомоза выполнена повторная операция. На следующий день у больного развилась гипотензия, которую коррегировали инфузионной терапией и кардиологическим медикаментозным лечением. У 1 (1\%) больного через 3 дня, у 1 (1\%) - через месяц после операции развилась дисфагия. После соответствую- щего консервативного лечения эндоскопически механического сужения не обнаружено. Через 3 мес состояние больного полностью нормализовалось. У 11 (10,5\%) больных через 14 мес выполнили абдоминопластику и подтяжку по их желанию.

Из 33 (37,7\%) больных, у которых до операции была гипертензия, у 22 давление нормализовалось. Из 12 (11,9\%) больных, страдающих гиперлипидемией, у 9 в последующие 3 мес эти нарушения исчезли. У 2 из 43 (41,3\%) пациентов с диабетом II типа уровни глюкозы в крови, значения НbA1с и C-пептида в первые 3 мес оставались повышенными, в более поздние сроки они снизились до нормального уровня. У 1 пациента дозу антидиабетического препарата снизили. У 13,6\% пациентов мужского пола обнаружены недостаточные уровни тестостерона в плазме крови и связывающего глобулина половых гормонов. Положительную динамику наблюдали у этих пациентов в течение 6 мес, до конца 12-го мес показатели полностью нормализовались, за исключением 1 пациента.

У 1 (1\%) больного через 5 дней после лСГ возникла несостоятельность швов в области дна желудка. Несостоятельность швов наблюдали у пациентов, которые прошли через LSQ. Больного немедленно госпитализировали и на фоне интенсивной терапии выполнили чрескожное дренирование подпеченочной и левой поддиафрагмальной областей. В последующие дни был установлен бариатрический стент. Через 3 дня пациент выписан на амбулаторное лечение. После 5-недельного динамического контроля стент удалили, последующий период протекал гладко.

У 11 (10,6\%) больных развился гастроэзофагеальный рефлюкс через 2 мес после ЛСГ, у 1 (1\%) - демпинг синдром. У 1 (1\%) больного после желудочного минишунтирования возникла диарея 5 - 6 раз в день. Всем больным в течение первых 90 дней после операции проводили лечение ингибиторами протонной помпы, кислотными нейтрализаторами и назначили диету.

За первые 6 мес у пациентов после стандартной ЛСГ уменьшилась масса тела в среднем на $(39,5 \pm 11,5)$ кг, у пациентов с наименьшим желудком - на (44 \pm 13$)$ кг, у пациентов после желудочного шунтирования - на (46 14) кг. В течение следующих 6 мес, как и в первые 6 мес, снижение массы тела было эффективным - на $(33,5 \pm$ $8,5)$ кг. Однако у больных после стандартной СлИВ гастрэктомии снижение массы тела в течение следующих 6 мес по сравнению с первыми 6 мес было менее эффективным - $(22,5 \pm 4,5)$ кг. У пациентов с резекцией антрума и сформированным меньшим желудком снижение массы тела было более эффективным - $(28,5 \pm 6,5)$ кг. Кроме того, в течение первых 3 мес больным после СлиВ гастрэктомии в отличие от больных после желудочного шунтирования не требовалась витаминно-минеральная поддержка. У пациентов после желудочного шунтирования анализы крови контролировали один раз в месяц, проводили парентерально соответствующее лечение витаминами и минералами. Через 12 мес после операции каждые 3 мес больные парентерально получали соответствующую витаминно-минеральную поддержку под контролем анализов крови. После всех операций масса тела за год снизилась на $(71,5 \pm 23,5)$ кг. 


\section{Обсуждение}

Гастроэзофагеальная рефлюксная болезнь после ЛСГ может быть вызвана нарушением кардиоэзофагеального угла во время операции и несоблюдением диеты после операции. Мы наблюдали эту патологию у 11 (10,6\%) больных. В мировой литературе есть данные о том, что эта болезнь встречается у $3-21 \%$ пациентов [6, 7].

Снижают эффективность лечения, проведенного пациентам, страдающим ожирением, психические расстройства. В публикациях, посвященных изучению взаимосвязи между гормональными и психическими расстройствами, сообщается, что 80\% пациентов с ожирением имеют симптомы биполярного расстройства [8]. Это не хирургическая проблема, однако у этих пациентов будет трудно добиться ожидаемого снижения массы тела, пока они будут испытывать чувство радости от еды, наслаждаться тайным питанием. Хотя до недавнего времени существовали критерии, по которым бариатрические процедуры признавали успешными или неудачными, многие исследователи считают, что снижение массы тела на 15 - 50\% - это успех [9].

Снижение массы тела в конце 12-го мес на 42,6\% показывает, что метод эффективен. При опросе 1 больного, у которого незначительно снизилась масса тела, выяснилось, что в периоды голодных кризов он употреблял слабоалкогольные напитки и высококалорийные продукты. После специальной диеты снижение массы тела вернулось в эффективное русло.

Основной причиной незначительного снижения массы тела после ЛСГ является недостаточная резекция. Развитие фистулы, стресс и депрессия, неправильный

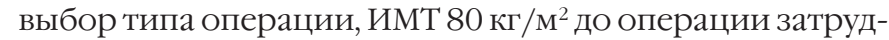
няют лечение сопутствующих заболеваний.

\section{Выводы}

1. Краткосрочные и среднесрочные результаты ЛСГ недостаточны для оценки этого метода, необходимы долгосрочные наблюдения.

2. В отличие от других методов, после ЛСГ отсутствует авитаминоз и эффективно снижается масса тела. Это способствует активному применению этого метода.

3. Применяемую нами новую методику ЛСГ, которая анатомически и физиологически наиболее близка к норме и после которой эффективно снижается ИМТ и устраняются сопутствующие заболевания, можно считать надежным методом для лечения ожирения, в том числе избыточного.

\section{Reaerences}

1. Arabi B, Olyaee M, Ranjbar E, Shiryazdi S, Shabahang H, Jangjoo A. Evaluation of laparoscopic sleeve gastrectomy compared with laparoscopic Roux-en-Y gastric bypass for people with morbid obesity: A systematic review and meta-analysis. Med J Islam Repub Iran, 2016;12(3):354-36. Published online 2016 Apr 12. PMID: 27453881.

2. Bariatric Today.(n.d.) Retrieved 15 November 2012. Available from: http://www.bariatrictoday.com/bariatric-surgery-information/themost-important-questions-to-ask-your-doctor.html

3. Buchwald H, Oien DM. Metabolic/bariatric surgery worldwide 2011. Obes Surg. 2013 Apr;23(4):427-36. doi: 10.1007/s11695-012-08640. PMID: 23338049

4. Dixon JB, Browne JL, Lambert GW, Jones KM, Reddy P, Pouwer F, et al. Severely obese people with diabetes experience impaired emotional well-being associated with socioeconomic disadvantage: results from diabetes MILES-Australia. Diabetes Res Clin Pract. 2013 Aug;101(2):131-40. doi: 10.1016/j.diabres.2013.05.017. PMID: 23806479. Epub 2013 Jun 24.

5. Eldar S, Heneghan HM, Brethauer SA, Schauer PR. Bariatric surgery for treatment of obesity. Int J Obes (Lond). 2011 Sep;35 Suppl 3:16-21. PMID: 21912381. doi: 10.1038/ijo.2011.142.

6. Khwaja H, Coelho A, Mazzarella M. et al. The IFSO Website (www.ifso. com): the Online Gateway to Obesity and Metabolic Disorders for Bariatric Surgery Professionals and Patients: On behalf of the IFSO Communications Committee. Obes Surg. 2015 Nov;25(11):2176-9. doi: 10.1007/s11695-015-1843-z. PMID: 26319793.

7. Lundell L. Principles and results of bariatric surgery. Dig Dis. 2012;30(2):173-7. doi: 10.1159/000336674. PMID: 22722434.

Epub 2012 Jun 20.

8. Schirmer B, Schauer PR. The surgical management of obesity. In: Brunicardi FC editor. Schwartz's Principles of Surgery. 9th ed. New York, NY: McGraw-Hill; 2010. Chapter 27. p. 949-978.

9. Terra X, Auguet T, Guiu-Jurado E, Berlanga A, Orellana-Gavaldà JM, Hernández M, et al. Long-term changes in leptin, chemerin and ghrelin levels following different bariatric surgery procedures: Roux-en-Y gastric bypass and sleeve gastrectomy. Obes Surg. 2013 Nov;23(11):1790-8. doi: 10.1007/s11695-013-1033-9. 